Institute, Imperial College \& Department of Respiratory Medicine, Royal Brompton NHS Foundation Trust, London, UK; ${ }^{6}$ Department of Respiratory Medicine, King's College Hospital, London, UK

Background Patients with Progressive Idiopathic Fibrotic Interstitial Lung Diseases (PIF-ILD) such as idiopathic pulmonary fibrosis have a short disease trajectory and have a similar prognosis to lung cancer patients. They have clear symptom control and quality of life (Qol) needs. The objective of this review was to evaluate the evidence for the use of pharmacological and non-pharmacological methods in improving dyspnoea, other symptoms and Qol for patients with PIF-ILD. In addition we assessed the use of outcome scales and economic evaluation of interventions.

Methods Studies were identified by searching eleven databases, relevant websites and hand searching key journals. Relevant studies were selected, assessed and data extracted independently by two researchers using standardised proformas. Meta-analyses were performed where appropriate and results presented as pooled mean difference with $95 \%$ CI. Effect sizes were also calculated where possible. A descriptive summary of other studies has been given.

Results 35 papers with 18 interventions were included. Metaanalyses were only possible for 3 interventions. Meta-analysis showed no significant treatment effect of IFN gamma $1 \mathrm{~b}$ or sildenafil on $6 \mathrm{MWD}$ or dyspnea. A positive treatment effect of pulmonary rehabilitation on 6MWD (effect size (95\% CI) 27.4 (4.1, 50.7) $\mathrm{p}=0.02$ ) was seen. Separate analysis showed a positive effect of pulmonary rehabilitation on dyspnea and a trend towards significant results for pulmonary rehabilitation and sildenafil in improving quality of life. There was weak evidence for the improvement of 6MWD using oxygen, dyspnea using prednisolone, diamorphine, $\mathrm{D}$-pencillamine and colchicine, cough using interferon alpha and thalidomide, anxiety using diamorphine, fatigue using pulmonary rehabilitation and Qol using thalidomide and doxycycline which warrants further research. There were a wide range of outcome scales used and no studies with economic evaluation.

Conclusion There is strong evidence for the use of pulmonary rehabilitation to improve $6 \mathrm{MWD}$ and moderate evidence for its use in improving dyspnoea and Qol. In addition, there is moderate evidence for sildenafil in improving Qol. There is weak evidence for a number of other interventions. Further research using economic evaluation and uniform outcome measures is needed.

\section{P132 DEFINING BENCHMARKS FOR CLINICAL OUTCOMES IN IDIOPATHIC PULMONARY FIBROSIS}

doi:10.1136/thoraxjnl-2012-202678.415

${ }^{1} \mathrm{AH}$ Cohen, ${ }^{1} \mathrm{WZ}$ Bradford, ${ }^{1} \mathrm{KF}$ Glassock, ${ }^{2} \mathrm{~F}$ Weber. 'InterMune Inc, Brisbane, USA, 2InterMune International AG, Muttenz, Switzerland

Introduction and Oobjectives Idiopathic pulmonary fibrosis (IPF) is a rare, fatal, progressive, fibrotic lung disorder that results in reduced lung capacity and has a considerable deleterious effect on patient function. To date, there has been no consensus on the magnitude of treatment effect that constitutes a clinically meaningful response to IPF therapy. Since IPF shares a range of biological and prognostic features with non-small cell lung cancer (NSCLC), we conducted a systematic review of clinical trials evaluating the efficacy of therapies for NSCLC to establish a benchmark for the treatment of IPF

Methods A literature search was performed to identify all randomised clinical trials between 1994-2010 evaluating therapies for NSCLC where a statistically significant effect of treatment on progression-free survival (PFS) or objective response rate (OR) was observed. The magnitude of the treatment effect in the NSCLC trials was compared to similar endpoints in three phase III clinical trials of pirfenidone in patients with IPF. In the NCSLC trials, PFS and OR were defined by standard conventions. In the IPF trials, PFS was defined as time to death or predefined thresholds for decline in forced vital capacity (FVC) or carbon monoxide diffusing capability. In the present analysis, objective response was defined according to predefined thresholds for change in FVC and the 6-minute walk test. Data were analysed according to the Cox proportional hazards model.

Results Twelve NCSLC trials, including a total of 13,959 patients, were identified by the search and included in the analysis. Of these studies, nine (12,456 patients) reported a significant effect on PFS, and seven (4,258 patients) reported a significant effect on OR. In both cases, the analysis showed that the magnitude of the response to therapy in the NSCLC trials was consistent with the pre-specified pirfenidone efficacy thresholds in the IPF trials.

Conclusion The clinical outcome parameters in therapeutic trials in NSCLC can be used to define benchmarks for assessing effect sizes in studies conducted in patients with IPF.

\section{P133 A SINGLE CENTRE RETROSPECTIVE SURVIVAL ANALYSIS OF PATIENTS WITH INTERSTITIAL LUNG DISEASE}

doi:10.1136/thoraxjnl-2012-202678.416

T Bradshaw, A Roychoudhury, SM Bianchi. Academic Department of Respiratory Medicine, Sheffield Teaching Hospital NHS Foundation Trust, Sheffield, UK

Introduction Idiopathic pulmonary fibrosis is a chronic, progressive condition. BTS guidelines indicate that the 'triple therapy' - Prednisolone, Azathioprine, N-acetylcysteine (NAC) - can be considered in the management of UIP (usual interstitial pneumonitis) pattern disease based on the outcome of the IFIGENIA study (Demedts NEJM 2005). Recent data from the PANTHER study (Raghu NEJM 2012) has led to reversal of this recommendation due to concerns of increased adverse events, deaths and hospitalisation in the triple therapy arm as compared with placebo controls and those receiving NAC alone. This retrospective review examines the survival outcome of patients with UIP and UIP/ COPD in a single centre (Sheffield Teaching Hospitals NHS Foundation Trust) where triple therapy was used over a five year period.

Methodology All patients with a UIP or UIP/COPD diagnosis managed within the interstitial lung disease clinic were identified through review of clinical notes and correspondence. Therapy, survival and demographic details were collected. Cox regression analysis was conducted using covariates of age at diagnosis, gender, occupational risk factors, COPD comorbidity and life limiting comorbidities.

Results 73 patients with UIP alone were identified; 16 patients had a UIP/COPD diagnosis. 8 patients received prednisolone alone, 2 NAC alone, 7 Pred/NAC, 3 Pred/Aza, 14 triple therapy and 11 received other immunosuppressive regimens (due to Azathioprine intolerance). 44 patients received no immunosuppressive therapy. Median survival, as derived from Kaplan-Meier curves, for those on no active therapy was 632 days (IQ range 485), 555 (IQ range 723) days for those on Prednisolone alone, 873 (IQ range 577)days for those on Pred/Aza and 869 (IO range 918) days for those on triple therapy. No increase in hospitalisation was noted in the triple therapy group as compared with 'untreated' patients.

Conclusions In a cohort of 89 patients treated over a 5 year period triple therapy has been associated with an improved survival as compared to any other treatment regimen. No increase in hospitalisation has been identified and serious untoward events including blood abnormalities have been rare and manageable. Further Cox analysis using the covariates discussed above allied with smoking history, FVC and TLCO at diagnosis and rate of decline in FVC and TLCO at 'decision to treat' as covariates is awaited. We hope this data may enlighten practitioners to the progression of UIP over time, the reasons adopted by clinicians in selection of various (often unproven) therapies and the safety and utility of these treatments in a real life population. 